\title{
Efficacy and Safety Study of Docetaxel as Salvage Chemotherapy in Metastatic Gastric Cancer Failing Fluoropyrimidine and Platinum Combination Chemotherapy
}

\author{
Jae-Lyun Lee, M.D. ${ }^{1}$, Min-Hee Ryu, M.D. ${ }^{1}$, Heung Moon Chang, M.D. ${ }^{1}$, Tae-Won Kim, M.D., Ph.D. ${ }^{1}$, Jeong \\ Hwan Yook, M.D., Ph.D. ${ }^{2}$, Sung Tae Oh, M.D., Ph.D. ${ }^{2}$, Byung Sik Kim, M.D., Ph.D. ${ }^{2}$, Jung Shin Lee, M.D., \\ Ph.D. ${ }^{1}$, and Yoon-Koo Kang, M.D., Ph.D. ${ }^{1}$ \\ ${ }^{1}$ Division of Oncology, Departments of Internal Medicine, ${ }^{2}$ Surgery, Asan Medical Center, University of Ulsan College of \\ Medicine, Seoul, Korea
}

Purpose: Fluoropyrimidine (F) and platinum (P) combination chemotherapy has been widely used for the first line treatment of advanced gastric cancer (AGC). Docetaxel (D) has shown promising activity in this disease. The present study retrospectively investigated the efficacy of $D$ monotherapy as salvage chemotherapy for AGC that is failing $F$ and $P$ combination chemotherapy.

Materials and Methods: A total of 34 patients, fitting the eligibility criteria, were included in this study. D was administered at a dose of $75 \mathrm{mg} / \mathrm{m}^{2}$ IV every 3 weeks, with dexamethasone prophylaxis. Twenty-nine patients had measurable lesions. The median treatment-free interval was 38.5 days, and $91.2 \%$ of patients had progressed within 4 months of withdrawal of the first line chemotherapy.

Results: A total of 133 cycles of D were administered, with a median of $3.5(1 \sim 8)$ cycles. From an intentionto-treat analysis, 6 patients achieved partial responses (PR), with a response rate of $20.7 \%(95 \% \mathrm{Cl}, 6.0 \sim 35.4)$.

\section{INTRODUCTION}

Gastric cancer is the fourth most common cancer and the second most common cause of cancer deaths in the world (1). According to a report of Korean Cancer Registry, gastric cancer was the most common cancer (24.0\%) and also the second leading cause of cancer deaths (18.7\%) during 2002 (2). Although improvements in early diagnosis have increased the number of curative resections, the main curative treatment

Correspondence: Yoon-Koo Kang, Department of Internal Medicine, University of Ulsan College of Medicine, Asan Medical Center, 388-1 Poongnap-dong, Songpa-gu, Seoul 138-736, Korea. (Tel) 82-2-3010-3230, (Fax) 82-2-3010-6961, (E-mail) ykkang@amc. seoul.kr

Received July 7, 2005, Accepted July 16, 2005

Presented in part at the $31^{\text {st }}$ Annual Meeting of Korean Cancer Association, Seoul, Korea, June 9 10, 2005.
The duration of objective PRs in these six were $2.3+, 2.5+$, $2.9,3.0+, 6.2$ and 6.8 months, respectively. Six patients showed a stable disease, but 15 showed progression. The median time to progression was 4.2 months $(95 \% \mathrm{Cl}, 2.8$ 5.5), with a median overall survival since the start of $D$ monotherapy of 8.4 months $(95 \% \mathrm{Cl}, 5.5 \sim 11.3)$. Grade $3 / 4$ neutropenia and febrile neutropenia occurred in $12.9 \%$ of patients and $3.1 \%$ of cycles. The incidence of grade 3 or worse non-hematological toxicities were as follows; peripheral sensory neuropathy $9.7 \%$, asthenia $3.2 \%$ and allergic reaction $2.7 \%$.

Conclusion: Docetaxel, $75 \mathrm{mg} / \mathrm{m}^{2}$, is active in AGC as second-line chemotherapy after failure of prior exposure to the $F$ and $P$ combination chemotherapy, with a favorable toxicity profile. (Cancer Res Treat. 2005;37:201-207)

Key Words: Stomach neoplasms, Docetaxel, Salvage therapy, Drug therapy

modality, many patients present with locally advanced unresectable or distant metastasis when first diagnosed. Even after complete resection, local and distant relapse are common. In these patients, palliative chemotherapy could improve the quantity and quality of life compared to best supportive care alone $(3 \sim 5)$.

5-fluorouracil (5-FU) and cisplatin have been widely used in different combinations, such as ECF (epirubicin, cisplatin, 5FU) and FUP (5-FU, cisplatin). In prospective randomized studies, ECF has led to significant response rates and survival benefit for patients with advanced gastric cancer compared with FAMTX (5-FU, adriamycin, high-dose MTX) chemotherapy (6, 7). Several nonrandomized studies demonstrated that superior response rates, up to $70 \%$, were achieved by combining cisplatin with 5 -FU in various schedules, with only moderately increased toxicity. In randomized phase III trials, FUP led to improved response rates compared with FAM (5-FU, doxorubicin and mitomycin) or 5-FU single-agent therapy (8), and showed a trend towards improved response rates compared with FAMTX, or ELF (etoposide, leucovorin and bolus 5-FU) (9). Therefore, 
FUP is now widely used as the first-line treatment for advanced gastric cancer. Recently, to relieve the inconvenience associate with intravenous infusion of 5-FU and improve safety, orally available 5-FU pro-drugs, such as capecitabine (10) and S-1 (11), are under active investigation and are currently used in clinical practice.

Unfortunately, about half the patients receiving chemotherapy are unresponsive, with the majority that achieved a response eventually having disease progression; the treatment results of second-line chemotherapy are also unsatisfactory. For these reasons, there is an urgent need for novel, active and less toxic regimens for patients with advanced gastric cancer who have failed first line chemotherapy incorporating fluoropyrimidine and platinum.

Docetaxel is a relatively new cytotoxic agent, which binds to, and stabilizes microtubules, causing cell-cycle arrest and apoptosis. The mechanism of action of docetaxel is different from fluoropyrimidine and platinum, which also lacks crossresistance between these agents. Docetaxel has shown singleagent activity in advanced gastric cancer. At a dose of 100 $\mathrm{mg} / \mathrm{m}^{2}$ every 3 weeks, docetaxel yielded response rates of $17 \sim$ $22 \%$ in chemotherapy-naïve $(12,13)$ and treated patients $(14$, 15). In these studies, the hematological toxicity was often significant, with about half of the patients experiencing grade 3 or worse neutropenia, which necessitated prophylactic growth factor support. In Asian studies $(16,17)$, docetaxel at a dose of $60 \sim 75 \mathrm{mg} / \mathrm{m}^{2}$ every 3 weeks produced a response rate of $16 \sim$ $24 \%$ in the first-line setting, with the similar frequencies of grade 3 hematological toxicities compared to those observed in the Western studies incorporating higher dose of docetaxel.

In this study, the efficacy and the safety of moderate dose $\left(75 \mathrm{mg} / \mathrm{m}^{2}\right)$ docetaxel, given 3-weekly, in patients with advanced gastric cancer who had failed first-line fluoropyrimidine and platinum chemotherapy, were retrospectively analyzed.

\section{MATERIALS AND METHODS}

\section{1) Patients}

A total of 48 patients with advanced gastric cancer were treated with docetaxel monotherapy as second-line chemotherapy, between March 2002 and November 2004, at the Asan Medical Center. Of these patients, subjects for the present study were selected if they fulfilled the following eligibility criteria: (1) histologically documented gastric or gastroesophageal junction adenocarcinoma; (2) prior exposure and failure to fluoropyrimidine (5-FU, capecitabine, doxifluridine, S1, or UFT) and platinum (cisplatin, heptaplatin, or oxaliplatin) chemotherapy; (3) Aged 18 to 70 years old; (4) ECOG performance status of 2 or lower; (5) no evidence of central nervous system metastases; (6) no significant cancer-related complications (i.e., intestinal obstruction and gastrointestinal bleeding); (7) adequate bone marrow and organ functions (WBCs $>4,000 / \mu 1$, absolute neutrophil count [ANC] $>1,500 / \mu 1$, hemoglobin $>9.0$ $\mathrm{g} / \mathrm{dl}$, and platelets $>100,000 / \mathrm{\mu l}$, creatinine $<1.5 \mathrm{mg} / \mathrm{dl}$, bilirubin $<1.5 \mathrm{mg} / \mathrm{dl}$, transaminases levels $<3$ times the upper normal limit); and (8) written informed consent to undergo docetaxel monotherapy. Patients with measurable lesions according to the RECIST criteria were preferred, but a measurable lesion was not prerequisite for the enrollment to assess the safety. The institutional review board of the Asan Medical Center granted permission for this retrospective study.

\section{2) Treatment protocol and dose adjustment}

Docetaxel, $75 \mathrm{mg} / \mathrm{m}^{2}$, was given as a 1 -hour intravenous infusion in $200 \mathrm{ml}$ of $5 \%$ dextrose water on day 1 every 3 weeks. Prophylaxis for potential docetaxel hypersensitivity was dexamethasone $8 \mathrm{mg}$ p.o. bid (or equivalent) for 3 days prior to starting docetaxel administration. The chemotherapy cycle was delayed if the granulocytes were $<1,500 / \mu l$ or if the platelet count did not return to a minimum of $100,000 / \mu 1$ on the day of infusion. Other non-hematological toxicities (except alopecia) were required to be either grade 0 or 1 before another cycle could be started.

During treatment docetaxel was reduced by $20 \%$ in patients with grade 4 neutropenia lasting for more than 7 days, febrile neutropenia, grade 4 thrombocytopenia or grade 3 thrombocytopenia with bleeding requiring a platelet transfusion, and grade 3 non-hematological toxicity, other than nausea, vomiting, anorexia and alopecia. Neither colony stimulating factors (CSFs) (e.g. G-CSF, GM-CSF) nor antibiotics were given prophylactically or to treat uncomplicated neutropenia. The cycles were continued for a maximum of six to eight cycles, unless disease progression, patients' refusal or unacceptable toxicity occurred.

\section{3) Efficacy and safety assessment}

The tumor response was evaluated according to the RECIST criteria: A complete response (CR) was defined as the disappearance of all evidence of cancer for 4 weeks or longer; A partial response (PR) as a $30 \%$ or more reduction in the sum of the longest diameters of target lesions for 4 weeks or longer; Stable disease as less than a $30 \%$ reduction or less than a $20 \%$ increase in the sum of the longest diameters of target lesions lasting 6 weeks or longer; Progressive disease as a more than $20 \%$ increase in the sum of the longest diameter of target lesions or the appearance of any new lesions. The tumor response was assessed every two to three treatment cycles. Confirmation of a response was carried out for those patients who showed PR or better after 2 or more cycles to demonstrate the persistence of the response. Toxicity was evaluated according to the NCI-CTCAE version 3.0.

\section{4) Statistics}

Descriptive statistics were reported as proportions and medians. The time to progression (TTP) was measured from the first day of docetaxel treatment until disease progression or the last day of the follow-up period without disease progression, and overall survival (OS) was measured from the first day of docetaxel treatment until death or the last day of the followup period. Kaplan-Meier estimates were used in the analysis of all time-event variables, with the differences between the curves were analyzed using the log-rank test. The dose intensity (DI) was calculated as the ratio of the total dose per square meter of the patients, divided by the total treatment duration expressed in weeks. The relative dose intensity was calculated as the ratio of the DI actually delivered to the DI planned by the protocol. SPSS for Windows (SPSS Inc., Chicago, IL) was used 
for statistical analyses.

\section{RESULTS}

\section{1) Patient characteristics}

Thirty-four of the 48 patients were enrolled in the present study. 14 patients were excluded from the study as they did not meet the eligibility criteria; 4 were older than 70 years, 10

Table 1. Baseline clinicopathological characteristics

\begin{tabular}{|c|c|c|}
\hline & No. of patients & $\%$ \\
\hline Total No. & 34 & 100 \\
\hline Age (median, range) & $53(31 \sim 70)$ & \\
\hline \multicolumn{3}{|l|}{ Measurable disease } \\
\hline Yes & 29 & 85.3 \\
\hline No & 5 & 14.7 \\
\hline \multicolumn{3}{|l|}{ Gender } \\
\hline Male & 29 & 85.3 \\
\hline Female & 5 & 14.7 \\
\hline \multicolumn{3}{|l|}{ ECOG performance status } \\
\hline 1 & 30 & 88.2 \\
\hline 2 & 4 & 11.8 \\
\hline \multicolumn{3}{|l|}{ Histology } \\
\hline Well/Moderately differentiated & 15 & 44.1 \\
\hline $\begin{array}{l}\text { Poorly differentiated or } \\
\text { signet-ring cell type }\end{array}$ & 19 & 55.9 \\
\hline \multicolumn{3}{|l|}{ Metastasis sites } \\
\hline Liver & 17 & 50.0 \\
\hline Peritoneum & 10 & 29.4 \\
\hline Lymph nodes & 23 & 61.8 \\
\hline Others* & 4 & 11.8 \\
\hline \multicolumn{3}{|l|}{ No. of metastatic sites } \\
\hline 1 & 18 & 52.9 \\
\hline 2 & 11 & 32.4 \\
\hline$\geq 3$ & 5 & 14.7 \\
\hline \multicolumn{3}{|l|}{ Disease status } \\
\hline Initially metastatic & 13 & 38.2 \\
\hline Recurrent & 21 & 61.8 \\
\hline \multicolumn{3}{|l|}{$\begin{array}{l}\text { Prior fluoropyrimidine and platinum } \\
\text { combination chemotherapy }\end{array}$} \\
\hline Capecitabine/Cisplatin & 17 & 50.0 \\
\hline 5-FU/Cisplatin & 11 & 32.4 \\
\hline Doxifluridine/Cisplatin & 4 & 11.8 \\
\hline S-1/Cisplatin & 1 & 2.9 \\
\hline 5-FU/Heptaplatin & 1 & 2.9 \\
\hline \multicolumn{3}{|l|}{ Additional chemotherapy exposure } \\
\hline Mitomycin C & 15 & 44.1 \\
\hline Irinotecan & 3 & 8.8 \\
\hline
\end{tabular}

*Other sites, include lung, bone, spleen and ovarian metastasis. had no prior exposure to platinum (2 of which were also older than 70 years) and 4 had inadequate bone marrow and organ functions ( 2 of which also had no prior exposure to platinum). Twenty-nine patients $(85.2 \%)$ had measurable lesions. The patients' baseline characteristics are listed in Table 1. The median age was 53 years (range, $31 \sim 70$ ). Including 24 patients (70.6\%), who had disease progression on previous chemotherapy, 31 patients $(91.2 \%)$ had disease progression within 4 months of withdrawal from prior fluoropyrimidine and platinum combination chemotherapy, and median time to progressive disease since the last day 1 of previous chemotherapy was 28 days (range, 7 369 days), with a median treatment-free interval of 38.5 days (range, $21 \sim 371$ days).

\section{2) Drug delivery}

The median number of chemotherapy cycles received was 3.5 (range, $1 \sim 8$; total 133 cycles). Treatment was delayed in 7 cycles $(5.3 \%)$ and dose reduction from the initial dose was executed in 3 patients. The reasons for dose reduction included febrile neutropenia in 3 patients and peripheral sensory neuropathy in 1 patient who also suffered from febrile neutropenia. The actual dose intensity was $23.9 \pm 2.2 \mathrm{mg} / \mathrm{m}^{2} /$ week, with a relative dose intensity of $95.6 \%$.

\section{3) Efficacy}

Six of the 29 patients with a measurable lesion achieved a confirmed PR as the best response, with a response rate of 20.7\% (95\% CI, 6.0 35.4). The durations of an objective response were $2.3+, 2.5+, 2.9,3.0^{+}, 6.2$ and 6.8 months, respectively. Another six patients had a confirmed SD, with an overall tumor control rate of $41.4 \%$. Responses were not assessable in 4 patients; three patients received only one cycle due to docetaxel-induced anaphylactic shock, aspiration pneumonia and death due to a traffic accident, respectively; one patient was lost to follow-up after 2 cycles of chemotherapy. At a median follow-up duration of 8.7 months for the surviving patients (range, $4.3 \sim 15.4$ months), the median time to progression (TTP)

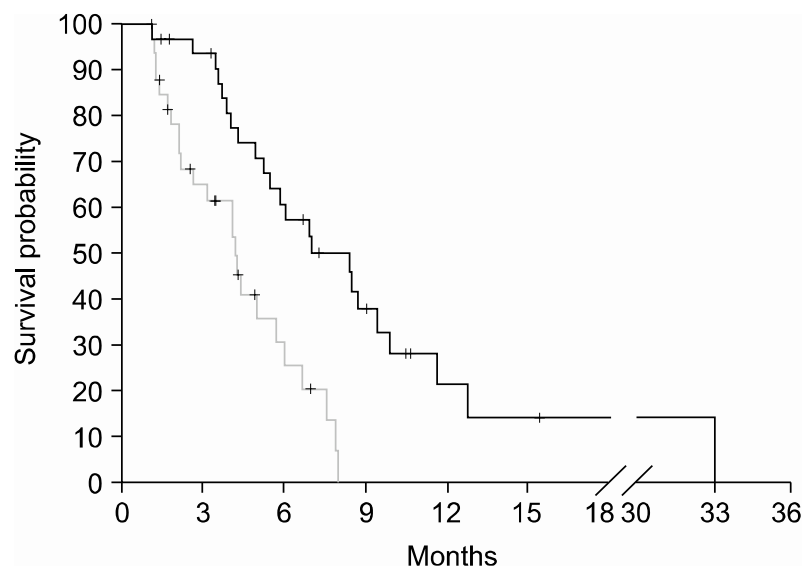

Fig. 1. Time to progression (gray solid line) and overall survival (black solid line) for the 34 patients with advanced gastric cancer treated with docetaxel monotherapy after having failed fluoropyrimidine and platinum combination chemotherapy. 
Table 2. Number of patients with toxicity attributable to docetaxel

\begin{tabular}{|c|c|c|c|c|c|}
\hline \multirow{2}{*}{ Category } & \multicolumn{5}{|c|}{ No. of patients $(\mathrm{N}=31)$} \\
\hline & 0 & 1 & 2 & 3 & 4 \\
\hline Anemia & $14(45.2 \%)$ & $8(25.8 \%)$ & $8(25.8 \%)$ & 1 & $0(3.2 \%)$ \\
\hline Leukopenia & $24(77.4 \%)$ & $3(9.7 \%)$ & $0(3.2 \%)$ & $1(9.7 \%)$ & 3 \\
\hline Granulocytopenia & $28(90.3 \%)$ & $1(3.2 \%)$ & 0 & 0 & $4(12.9 \%)$ \\
\hline Thrombocytopenia & $29(93.5 \%)$ & $2(6.5 \%)$ & 0 & 0 & 0 \\
\hline Febrile neutropenia & $28(90.3 \%)$ & & & $3(9.7 \%)$ & $1(3.2 \%)$ \\
\hline Asthenia & $3(9.7 \%)$ & $5(16.1 \%)$ & $22(71.0 \%)$ & $1(3.2 \%)$ & 0 \\
\hline Anorexia & $23(74.2 \%)$ & $4(12.9 \%)$ & $4(12.9 \%)$ & 0 & 0 \\
\hline Nausea & $26(83.9 \%)$ & $5(16.1 \%)$ & 0 & 0 & 0 \\
\hline Stomatitis & $13(41.9 \%)$ & $12(38.7 \%)$ & $6(19.4 \%)$ & 0 & 0 \\
\hline Diarrhea & $27(87.1 \%)$ & $3(9.7 \%)$ & $1(3.2 \%)$ & 0 & 0 \\
\hline Dacryorrhoea & $29(93.5 \%)$ & $1(3.2 \%)$ & $1(3.2 \%)$ & 0 & 0 \\
\hline Sensory neuropathy & $16(51.6 \%)$ & $9(29.0 \%)$ & $3(9.7 \%)$ & $3(9.7 \%)$ & 0 \\
\hline Motor neuropathy & $27(87.1 \%)$ & $3(9.7 \%)$ & $1(3.2 \%)$ & 0 & 0 \\
\hline Nail change & $13(41.9 \%)$ & $15(48.4 \%)$ & $3(9.7 \%)$ & 0 & 0 \\
\hline Edema & $18(48.6 \%)$ & $8(25.8 \%)$ & $5(16.1 \%)$ & 0 & 0 \\
\hline Allergic reaction* & $30(93.8 \%)$ & $1(2.7 \%)$ & 0 & $1(2.7 \%)$ & 0 \\
\hline
\end{tabular}

*Available in 32 patients.

Table 3. Number of cycles with toxicity attributable to docetaxel

\begin{tabular}{lrcccc}
\hline \multicolumn{1}{c}{ Category } & \multicolumn{3}{c}{ No. of cycles (N=127) } \\
\cline { 2 - 6 } & 0 & 1 & 2 & 3 & 4 \\
\hline Anemia & $77(60.6 \%)$ & $34(26.8 \%)$ & $15(11.8 \%)$ & $1(0.8 \%)$ & 0 \\
Leukopenia & $119(93.7 \%)$ & $4(3.1 \%)$ & 0 & $1(0.8 \%)$ & $3(2.4 \%)$ \\
Granulocytopenia & $122(96.1 \%)$ & $1(0.8 \%)$ & 0 & 0 & $4(3.1 \%)$ \\
Thrombocytopenia & $125(98.4 \%)$ & $2(1.6 \%)$ & 0 & 0 & 0 \\
Febrile neutropenia & $123(96.9 \%)$ & & & $3(2.4 \%)$ & $1(0.8 \%)$ \\
Asthenia & $32(25.2 \%)$ & $48(37.8 \%)$ & $46(36.2 \%)$ & $1(0.8 \%)$ & 0 \\
Anorexia & $111(87.4 \%)$ & $12(9.4 \%)$ & $4(3.1 \%)$ & 0 & 0 \\
Nausea & $122(96.1 \%)$ & $5(3.9 \%)$ & 0 & 0 & 0 \\
Stomatitis & $93(73.2 \%)$ & $5(3.9 \%)$ & $1(0.8 \%)$ & 0 & 0 \\
Diarrhea & $121(95.3 \%)$ & $5(3.9 \%)$ & $1(0.8 \%)$ & 0 & 0 \\
Dacryorrhoea & $119(93.8 \%)$ & $4(3.1 \%)$ & $4(3.1 \%)$ & 0 & 0 \\
Sensory neurotoxicity & $69(54.3 \%)$ & $39(30.7 \%)$ & $4(11.0 \%)$ & $5(3.9 \%)$ & 0 \\
Motor neurotoxicity & $119(93.7 \%)$ & $6(4.7 \%)$ & $2(1.6 \%)$ & 0 & 0 \\
Nail change & $64(50.4 \%)$ & $59(46.5 \%)$ & $4(3.1 \%)$ & 0 & 0 \\
Edema & $90(70.9 \%)$ & $26(20.5 \%)$ & $11(8.7 \%)$ & 0 & 0 \\
Allergic reaction* & $126(99.2 \%)$ & $1(0.8 \%)$ & 0 & $1(0.8 \%)$ & 0 \\
\hline
\end{tabular}

*Available in 128 cycles.

and overall survival (OS) were 4.2 months (95\% CI, $2.8 \sim 5.5$, Fig. 1) and 8.4 months (95\% CI, 5.5 11.3), respectively. The Median TTP and OS for patients with measurable lesions were not different from those of the whole population; 4.2 months for the TTP $(95 \% \mathrm{CI}, 2.8 \sim 5.5)$ and 8.5 months for the OS (95\% CI, 5.3 11.6). No differences in the TTP and OS were observed according to age ( $\leq 53$ vs. $>53$ ), sex, histological differentiation, number of metastatic sites and TTP in the first-line setting. Patients with a prior response to previous chemotherapy (PR or better vs. SD or worse, $p=0.053$ ) and longer treatment-free interval $(\leq 30$ days vs. $>30$ days, $p=$ 0.08 ) had a tendency to have prolonged OS. 


\section{4) Toxicity}

For each toxicity, the patient distribution, with respect to highest observed grade, is summarized in Table 2. The incidence of adverse events per chemotherapy cycle is listed in Table 3. The most common hematological toxicity was mild or moderate anemia. Grade 3 or worse leukopenia or granulocytopenia occurred in 4 patients $(12.9 \%)$. There was only 1 patient who had grade 1 or 2 granulocytopenia. Four patients developed febrile neutropenia, which were treated successfully with antibiotics and G-CSF. There were no episodes of grade 2 or worse thrombocytopenia or clinical bleeding. For grade 2 or worse non-hematological toxicities, asthenia was most frequently seen $(74.2 \%)$ followed by stomatitis (19.4\%), peripheral sensory neuropathy (19.4\%), fluid retention (16.1\%), anorexia $(12.9 \%)$ and nail toxicity $(9.7 \%)$. However, clinically relevant grade 3 or worse non-hematological adverse events were limited to peripheral sensory neuropathy $(9.7 \%)$ and asthenia $(3.2 \%)$. Within 30 days of the last dose of chemotherapy one patient died due to aspiration pneumonia, but she was not neutropenic. The causative relationship of her death due to docetaxel chemotherapy remained speculative and unconfirmed.

\section{DISCUSSION}

Fluoropyrimidine and platinum combination chemotherapy, with or without anthracycline, is one of the most commonly used regimen as the first-line treatment in advanced gastric cancer $(7 \sim 11)$. Although it produce a response rate of approximately $30 \sim 70 \%$, most patients eventually have disease progression, irrespective of the initial response to first-line chemotherapy, and the second-line chemotherapy in these patients, especially for those who have a good performance status, remains a challenge. Newer agents, such as docetaxel, iriotecan and oxaliplatin, have been investigated in this setting. Thirtyfour consecutive patients with advanced gastric cancer, treated with docetaxel monotherapy as second-line regimen after having failed fluoropyrimidine and platinum combination chemotherapy, were retrospectively analyzed in the study.

Docetaxel has shown single-agent activity in advanced gastric cancer. In a second-line setting, Giuliani et al. (14) reported a $17 \%$ response rate with single-agent docetaxel, $100 \mathrm{mg} / \mathrm{m}^{2}$, in 30 patients with advanced gastric cancer refractory to ECF or PELF (cisplatin, epirubicin, leucovorin, 5-FU). Vanhoefer et al. (15) reported a $20 \%$ response rate with docetaxel, $100 \mathrm{mg} /$ $\mathrm{m}^{2}$, in 25 patients that had already been exposed to first-line FUP. Andre et al. (18) found a $21 \%$ response rate when 25 patients pretreated with FUP were given epirubicin, $75 \mathrm{mg} / \mathrm{m}^{2}$, plus docetaxel, $75 \mathrm{mg} / \mathrm{m}^{2}$, every 3 weeks. Our results demonstrate that docetaxel given 3-weekly, at a dose of $75 \mathrm{mg} / \mathrm{m}^{2}$ is feasible with moderate activity against fluoropyrimidine and platinum refractory gastric cancer in general clinical practice. A response rate of $20.7 \%$, with a disease control rate of $41.4 \%$, in this study was largely consistent with previous reports using $100 \mathrm{mg} / \mathrm{m}^{2}$ docetaxel $(14,15)$ or the docetaxel and epirubicin combination regimen as second-line salvage (18). In addition, the response rate achieved in this study was comparable to those achieved in chemotherapy-naive patients $(12,13,16)$. Bang et al. (16) reported a $16 \%$ response rate with docetaxel, $75 \mathrm{mg} /$ $\mathrm{m}^{2}$, in 45 chemotherapy-naïve patients. Einzig et al. (13) reported a $17 \%$ response rate with docetaxel, $100 \mathrm{mg} / \mathrm{m}^{2}$, in 41 patients. Sulkes et al. (12) found a $24 \%$ response rate when 33 patients were given docetaxel, $100 \mathrm{mg} / \mathrm{m}^{2}$, every 3 weeks in a first line setting. The response rate in the current study was also comparable to those reported using weekly irinotecan (19), modified FOLFIRI $(20,21)$ or modified FOLFOX chemotherapy (22), which required weekly outpatient visits or intravenous 5-FU infusion.

The median TTP and OS were 4.2 and 8.4 months, respectively. The TTP and OS of the current study were comparable, or somewhat better, to the published data from most secondline chemotherapy trials using docetaxel at a higher dosage $(14,15)$, irinotecan $(19 \sim 21)$ and oxaliplatin $(22)$, where fluoropyrimidine and platinum refractory gastric cancer patients were included as study subjects. The median TTP was also comparable to that achieved using docetaxel, $75 \mathrm{mg} / \mathrm{m}^{2}$, in a first line setting (16). However, this may reflect the fact that the proportion of patients with recurrent diseases, which might have less tumor burden and longer survival (23), were higher than those reported in previous studies.

Our results showed the toxicity was modest in intensity, with grade 2 asthenia representing the leading toxicity, which was observed in $71 \%$ of patients. The second most common grade 2 or worse toxicities observed were stomatitis (19.4\%) and peripheral sensory neuropathy $(19.4 \%)$, followed by edema $(16.1 \%)$, anorexia $(12.9 \%)$ and nail toxicity $(9.7 \%)$. Although hematological toxicity was reported, the incidences of grade 3 or worse leukopenia or granulocytopenia were approximately $10 \%$ per patient and $3 \%$ per cycle. Febrile neutropenia developed in 4 patients: grade 3 in 3 patients $(9.7 \%)$ and grade 4 in $1(3.2 \%)$, which developed without G-CSF prophylaxis, and were managed successfully with antibiotics and G-CSF. Febrile neutropenia developed in the first cycle of chemotherapy in 3 patients, and in 1 further patient during the third cycle, but did not recur after adequate dose reduction. There were no treatment-related deaths with direct causal relationship. The incidence of grade 3 4 neutropenia in the current study was somewhat less than those shown by Vanhoefer (15), and comparable to those reported by Giuliania (14), which incorporated prophylactic G-CSF in the treatment protocol. The toxicity profile appears to be more favorable compared to those observed in studies using irinotecan-based chemotherapy $(19,20)$. However, caution should be used when comparing the hematological toxicities between studies, as the hematological monitoring strategies could be different. In the current study, $\mathrm{CBC}$ was repeated before each chemotherapy cycle, while it was repeated every week for the first two cycles in the study conducted by Bang et al. (16), which reported grade 3 or worse neutropenia in $81.8 \%$ of the patients and $36.1 \%$ of the cycles with the same docetaxel dosage.

Based on comparable activity and favorable safety profiles to other second-line regimens, $(14,15,19 \sim 22)$ the docetaxel monotherapy, at a dose of $75 \mathrm{mg} / \mathrm{m}^{2}$, might be considered a promising second-line chemotherapy in fluoropyrimidine- and platinum-refractory advanced gastric cancers. There have been no randomized trials comparing the efficacy and safety of the docetaxel versus irinotecan- or oxaliplatin-containing regimen 
in a second-line setting. Further efforts are needed to clarify the optimal salvage regimen.

Recently, the TAX325 study group (24) reported superior efficacy results with respect to response rates, TTP and OS could be achieved by adding docetaxel to FUP (DCF) compared with FUP combination chemotherapy in a first line setting: the response rates were $37 \%$ vs. $25 \%(\mathrm{p}=0.0106)$ and the TTP 5.6 months vs. 3.7 months $(\mathrm{p}<0.01)$, respectively. However, DCF caused significant hematological toxicity, including grade 3 or worse neutropenia in $82.3 \%$ and febrile neutropenia in $30.3 \%$ of patients treated with DCF, which required the secondary prophylactic use of G-CSF. Although the risk of death was reduced in the triplet ( $\mathrm{p}=0.0201$ ), with median OS of 9.2 months vs. 8.6 months compared to the doublet, the survival difference seems to have no clinical significance. Given its modest benefit in survival, increased toxicity profile and incurable disease status, it is not known which is better to incorporate with docetaxel in a first-line triplet or to reserve for the second-line regimen following first-line chemotherapy failure. Our group also reported on the efficacy and safety profiles of the capecitabine plus cisplatin (XP) (10) and XP with the docetaxel triplet (DXP) (25) regimens. Treatment outcomes of the XP regimen have been comparable to those of the FUP regimen, but with a more favorable toxicity profile, which can be used conveniently on an outpatient clinic basis. In our phase I/II study, the DXP regimen seems to be more active than the XP regimen. However, although generally well tolerated, the DXP regimen was more toxic than the XP regimen. Generally, sequential chemotherapy is expected to be more tolerable than concurrent triplet chemotherapy. Moreover, treatment outcomes, especially overall survival, can be improved by effective second-line chemotherapy. In this study, docetaxel showed good activity as a salvage second-line chemotherapy in advanced gastric cancer, which was in line with previous reports. Further well-designed, prospective randomized trials are needed to clarify whether the addition of docetaxel to FUP or XP will provide superior benefit compared with the sequential administration of the same agents (FUP or XP followed by docetaxel) in the treatment of advanced gastric cancer.

There were several limitations in this study. The first is the inherent selection bias and potential data imperfection of retrospective study design. However, the eligibility criteria were established before data collection and approximately $60 \%$ of the subjects were enrolled on another prospective study on docetaxelinduced toxicity, which enabled us to gather reliable data on toxicities. The second is that patients with both measurable and non-measurable disease were entered into this study, which made response evaluation slightly more difficult, but also allowed us to evaluate the safety profile in more patients.

\section{CONCLUSIONS}

The docetaxel monotherapy, at a dose of $75 \mathrm{mg} / \mathrm{m}^{2}$, is a valuable regimen as a second-line treatment in patients with advanced gastric cancer having failed prior fluoropyrimidine and platinum combination chemotherapy. The favorable safety profile, with conserved activity, led us to consider further evaluation of this regimen in the context of a prospective phase II trial in this patient population, for which patients are open and actively being enrolled.

\section{REFERENCES}

1. Parkin DM, Bray F, Ferlay J, Pisani P. Global Cancer Statistics, 2002. CA Cancer J Clin. 2005;55:74-108.

2. Shin HR, Jung KW, Won YJ, Park JG. 2002 annual report of the Korea Central Cancer Registry: based on registered data from 139 hospitals. Cancer Res Treat. 2004;36:103-14.

3. Murad AM, Santiago FF, Petroianu A, Rocha PR, Rodrigues MA, Rausch M. Modified therapy with 5-fluorouracil, doxorubicin, and methotrexate in advanced gastric cancer. Cancer. 1993;72:37-41.

4. Glimelius B, Ekstrom K, Hoffman K, Graf W, Sjoden PO, Haglund $\mathrm{U}$, et al. Randomized comparison between chemotherapy plus best supportive care with best supportive care in advanced gastric cancer. Ann Oncol. 1997;8:163-8.

5. Pyrhonen S, Kuitunen T, Nyandoto P, Kouri M. Randomised comparison of fluorouracil, epidoxorubicin and methotrexate (FEMTX) plus supportive care with supportive care alone in patients with non-resectable gastric cancer. Br J Cancer. 1995; 71:587-91.

6. Waters JS, Norman A, Cunningham D, Scarffe JH, Webb A, Harper P, et al. Long-term survival after epirubicin, cisplatin and fluorouracil for gastric cancer: results of a randomized trial. Br J Cancer. 1999;80:269-72.

7. Webb A, Cunningham D, Scarffe JH, Harper P, Norman A, Joffe JK, et al. Randomized trial comparing epirubicin, cisplatin, and fluorouracil versus fluorouracil, doxorubicin, and methotrexate in advanced esophagogastric cancer. J Clin Oncol. 1997;15:261-7.

8. Kim NK, Park YS, Heo DS, Suh C, Kim SY, Park KC, et al. A phase III randomized study of 5-fluorouracil and cisplatin versus 5-fluorouracil, doxorubicin, and mitomycin $\mathrm{C}$ versus 5-fluorouracil alone in the treatment of advanced gastric cancer. Cancer. 1993;71:3813-8.

9. Vanhoefer U, Rougier P, Wilke H, Ducreux MP, Lacave AJ, Van Cutsem E, et al. Final results of a randomized phase III trial of sequential high-dose methotrexate, fluorouracil, and doxorubicin versus etoposide, leucovorin, and fluorouracil versus infusional fluorouracil and cisplatin in advanced gastric cancer: a Trial of the European Organization for Research and Treatment of Cancer Gastrointestinal Tract Cancer Cooperative Group. J Clin Oncol. 2000;18:2648-57.

10. Kim TW, Kang YK, Ahn JH, Chang HM, Yook JH, Oh ST, et al. Phase II study of capecitabine plus cisplatin as first-line chemotherapy in advanced gastric cancer. Ann Oncol. 2002; 13:1893-8.

11. Koizumi W, Tanabe S, Saigenji K, Ohtsu A, Boku N, Nagashima F, et al. Phase I/II study of S-1 combined with cisplatin in patients with advanced gastric cancer. Br J Cancer. 2003; 89:2207-12.

12. Sulkes A, Smyth J, Sessa C, Dirix LY, Vermorken JB, Kaye $\mathrm{S}$, et al. Docetaxel (Taxotere) in advanced gastric cancer: results of a phase II clinical trial. EORTC Early Clinical Trials Group. Br J Cancer. 1994;70:380-3.

13. Einzig AI, Neuberg D, Remick SC, Karp DD, O'Dwyer PJ, Stewart JA, et al. Phase II trial of docetaxel (Taxotere) in patients with adenocarcinoma of the upper gastrointestinal tract previously untreated with cytotoxic chemotherapy: the Eastern Cooperative Oncology Group (ECOG) results of protocol E1293. Med Oncol. 1996;13:87-93.

14. Giuliani F, Gebbia V, De Vita F, Maiello E, Di Bisceglie M, Catalano $\mathrm{G}$, et al. Docetaxel as salvage therapy in advanced gastric cancer: a phase II study of the Gruppo Oncologico 
Italia Meridionale (G.O.I.M.). Anticancer Res. 2003;23(5b): 4219-22.

15. Vanhoefer U, Wilke H, Harstrick A, Achterrath W, Preusser $\mathrm{P}$, Stahl M, et al. Phase II study of docetaxel as second line chemotherapy in metastatic gastric cancer. Proc Am Soc Clin Oncol. 1999;18:303(abstr).

16. Bang YJ, Kang WK, Kang YK, Kim HC, Jacques C, Zuber E, et al. Docetaxel $75 \mathrm{mg} / \mathrm{m}^{2}$ is active and well tolerated in patients with metastatic or recurrent gastric cancer: a phase II trial. Jpn J Clin Oncol. 2002;32:248-54.

17. Taguchi T, Sakata Y, Kanamaru R, Kurihara M, Suminaga M, Ota J, et al. Late phase II clinical study of RP56976 (docetaxel) in patients with advanced/recurrent gastric cancer: a Japanese Cooperative Study Group trial (group A). Gan To Kagaku Ryoho. 1998;25:1915-24.

18. Andre T, Louvet C, Ychou M, Gamelin E, Mousseau M, Carola $\mathrm{E}$, et al. Docetaxel-epirubicin as second-line treatment for patients with advanced gastric cancer. Proc Am Soc Clin Oncol. 1999;18:1062(abstr)

19. Chun JH, Kim HK, Lee JS, Choi JY, Lee HG, Yoon SM, et al. Weekly irinotecan in patients with metastatic gastric cancer failing cisplatin-based chemotherapy. Jpn J Clin Oncol. 2004; 34:8-13.

20. Assersohn L, Brown G, Cunningham D, Ward C, Oates J, Waters JS, et al. Phase II study of irinotecan and 5-fluorouracil/leucovorin in patients with primary refractory or relapsed advanced oesophageal and gastric carcinoma. Ann Oncol. 2004; 15:64-9.

21. Kim ST, Kang WK, Kang JH, Park KW, Lee J, Lee SH, et al. Salvage chemotherapy with irinotecan, 5-fluorouracil and leucovorin for taxane- and cisplatin-refractory, metastatic gastric cancer. Br J Cancer. 2005;92:1850-4.

22. Kim DY, Kim JH, Lee SH, Kim TY, Heo DS, Bang YJ, et al. Phase II study of oxaliplatin, 5-fluorouracil and leucovorin in previously platinum-treated patients with advanced gastric cancer. Ann Oncol. 2003;14:383-7.

23. Lee SS, Lee JL, Ryu MH, Chang HM, Kim TW, Kim WK, et al. Combination chemotherpy with capecitabine $(\mathrm{X})$ and cisplatin $(\mathrm{P})$ as a first line treatment of advanced gastric cancer: experience of 246 patients with prognostic factor analysis. Proc ECCO 13 2005:(submitted).

24. Moiseyenko VM, Ajani JA, Tjulandin SA, Majlis A, Constenla $\mathrm{M}$, Boni $\mathrm{C}$, et al. Final results of a randomized controlled phase III trial (TAX 325) comparing docetaxel (T) combined with cisplatin $(\mathrm{C})$ and 5-fluorouracil $(\mathrm{F})$ to $\mathrm{CF}$ in patients (pts) with metastatic gastric adenocarcinoma (MGC). Proc Am So Clin Oncol. 2005;23:308s(abstr 4002).

25. Kang YK, Kim TW, Chang HM, Ryu MH, Yook JH, Oh ST, et al. A phase I/II trial of docetaxel, capecitabine, and cisplatin as a first line chemotherapy for advanced gastric cancer. Proc Am So Clin Oncol. 2004;22:(abstr 4066). 